\title{
Optimal bladder diaryduration for patients with suprapontine neurogenic lower urinary tract dysfunction
}

\author{
Charalampos Konstantinidis ${ }^{1}$, Zisis Kratiras ${ }^{2}$, Michael Samarinas ${ }^{3}$, Konstantinos Skriapas ${ }^{2}$ \\ ${ }^{1}$ Urology \& Neuro-urology Unit, National Rehabilitation Center, Athens, Greece; ${ }^{2}$ Department of \\ Urology, General Hospital of Larissa "Koutlibanio", Larissa, Greece; ${ }^{3}$ Department of Urology, University \\ of Thessaly, Larissa, Greece
}

\section{ABSTRACT}

Purpose: To identify the minimum bladder diary's length required to furnish reliable documentation of LUTS in a specific cohort of patients suffering from neurogenic urinary dysfunction secondary to suprapontine pathology.

Materials and Methods: From January 2008 to January 2014, patients suffering from suprapontine pathology and LUTS were requested to prospectively complete a bladder diary form for 7 consecutive days. Micturitions per day, excreta per micturition, urgency and incontinence episodes and voided volume per day were evaluated from the completed diaries.

We compared the averaged records of consecutive days (2-6 days) to the total 7 days records for each patient's diary, seeking the minimum diary's length that could provide records comparable to the 7 days average, the reference point in terms of reliability. Results: From 285 subjects, 94 male and 69 female patients enrolled in the study. The records of day 1 were significantly different from the average of the 7 days records in every parameter, showing relatively small correlation and providing insufficient documentation. Correlations gradually increased along the increase in diary's duration. According to our results a 3-day duration bladder diary is efficient and can provide results comparable to a 7 day length for four of our evaluated parameters. Regarding incontinence episodes, 3 days seems inadequate to furnish comparable results, showing a borderline difference.

Conclusions: A 3-day diary can be used, as its reliability is efficient regarding number of micturition per day, excreta per micturition, episodes of urgency and voided volume per day.

\section{ARTICLE INFO}

\section{Keywords:}

Urinary Bladder; Pathology;

Neurogenic Bowel; Lower

Urinary Tract Symptoms

Int Braz J Urol. 2016; 42: 766-72

Submitted for publication:

May 31, 2015

Accepted after revision:

October 12, 2015

\section{INTRODUCTION}

Lower Urinary Tract Symptoms (LUTS) secondary to neurological disorders appear to be a major health problem affecting patients worldwide (1). Voiding dysfunction has a remarkably negative impact on the quality of life and may lead to significant and life threatening complications (2), increasing simultaneously the economic burden for patients and health providers. The usual history-taking in patients with neurological disorders seems inadequate for the assessment of LUTS due to subjective factors. Several clinical trials targeting a more objective and accurate evaluation of LUTS have employed urinary diaries in order to identify voiding patterns and to quantify response to treatment. According to the International Continence Society, three types of urinary diaries 
have been defined (3): Micturition Chart, Frequency Volume Chart and Bladder Diary (BD).

Nevertheless, a validated standardized bladder diary in terms of diary format, duration and evaluated parameters, has not yet been established (4). As a result, a BD with an optimum duration, which may ensure reliable results having simultaneously an acceptable level of patient's compliance, remains a dispute.

Recent and former literature has studied mostly non-neurogenic populations, having only rare references focusing on neurogenic lower urinary tract dysfunction in patients suffering from suprapontine pathology. So, the aim of our study was to determine the optimal length of a BD in terms of test reliability in this special cohort of patients. Analyzing the data derived from a 7-day bladder diary we evaluated five parameters seeking the optimal length for each one, assuming that the 7-day duration urinary diary is the reference point in the assessment of LUTS $(5,6)$.

\section{MATERIALS AND METHODS}

Type of the study

Prospective, quantitative survey from January 2008 to January 2014, involving 285 consecutive patients.

\section{Inclusion/exclusion criteria}

Inclusion criteria were treatment-naive patients of both genders, older than 20 years, suffering from suprapontine pathology caused either by traumatic brain injury or by cerebrovascular accident (CVA). Patients should be mentally fit with the ability to understand and communicate and the appearance of their LUTS should prelude at least 2 weeks before the integration on this study. For the mental evaluation of our subjects, Mini Mental State Examination was used (7). Exclusion criteria were multiple sclerosis, confused state, depression, dementia, bladder cancer, pregnancy, bladder stones, bladder outlet obstruction, diabetes, and urinary tract infection. Furthermore, subjects with a previous history of stress incontinence were also excluded from this study, in order to avoid the real cause of present LUTS to be confused and mixed. Additionally, male individuals over 50 years of age were excluded, considering that the co-existing benign prostate hyperplasia might interfere and distort our results. Moreover, after a proper vaginal examination, female patients suffering from any kind of vaginal prolapse were also excluded from the study, since vaginal prolapse might cause bladder outlet obstruction distorting our results.

\section{Process}

All patients at the initial visit were clinically evaluated with physical examination, history report, urine analysis and urine culture, abdominal ultrasound and post-void residual volume evaluation. Patients received a bladder diary form and were requested to complete it for 7 consecutive days beginning with the first morning void. Patients were advised to record the precise time and volume of each void. The beginning of every day was defined at 08.00a.m. and the end at 07.59a.m. next morning (8).

Additionally, the episodes of urgency and incontinence have also been recorded. The evaluated parameters of our study were: micturitions per day (voiding frequency), excreta per micturition (mL per void), episodes of urgency and incontinence and voided volume per day.

\section{Statistical analysis}

At the second visit, we analyzed the diaries and averaged the records over 2 to 7 days. The records of each subject were considered as dependent scale parameters and the total average of the 7 days records as the reference point of our comparisons in terms of test reliability.

As a result we used paired t-tests, in order to compare the averaged records of different days (2-6 days) to the total 7 days records for each patient's diary. We sought to identify which day's mean had no statistically significant difference with the 7 days average in terms of each assessed parameter. In all comparisons $p$ value $<0.05$ was considered as statistically significant. SPSS version 20.0 was used for the statistical analysis. 


\section{RESULTS}

From the total of 285 patients, 122 (42\%) patients were excluded while 163 subjects (58\%) met the inclusion criteria and were prospectively enrolled in the study. Among the 122 patients, 12 (9\%), 31 (25\%) and 30 (24\%) were excluded from the study due to urinary infection, diabetes and mental instability respectively. In addition, 40 $(32 \%)$ male patients over 50 years old were also excluded. The 94 male and 69 female patients enrolled in our study ranged in age from 23 to 68 years, with a mean age of 43.6 years.

The data retrieved from the diaries were summarized, averaged and the descriptive statistics along with the general characteristics of our cohort are provided in Tables 1a and 1b. Correlations gradually increased along the increase in the diary's duration reaching the maximum on the day 6 . Table- 2 summarizes the correlations of the different diary's duration records with the 7 days average.
According to our test results, a 1-day and 2-day length bladder diary could not provide results comparable to a 7-day diary in any parameter, since the differences that arose using the paired t-test between these durations were statistically significant. Thus, regarding micturitions per day, excreta per micturition, urgency episodes and total voided volume the 3-day records were not significantly different than the 7 days records ( $>>0.05$ ). This absence of statistical significance was interpreted as a failure to establish a true difference between the 3-day length and the 7-day length records for 4 of the 5 evaluated parameters. As a result, the 3-day diary could provide data comparable to the 7-days in terms of these parameters. As for the incontinence episodes the 3-days length was borderline significantly different from the 7-day length $(\mathrm{p}=0.044)$, becoming statistically non significant from day 4 and on. Table- 3 summarizes the results of the comparisons between the different diary's lengths. Comparisons between 1-day to 7-day and

Table 1a - Descriptive statistics of the cohort. Average data derived from the completed diaries.

\begin{tabular}{lcccc}
\hline & Number of patients & Mean & Std. Error & Std. Deviation \\
\hline Av. Micturitions & 163 & 9.92 & 0.19 & 2.44 \\
Av. Excreta/micturition (mL/void) & 163 & 121.83 & 1.45 & 18.44 \\
Av. Urgency Incidents & 163 & 5.10 & 0.06 & 0.73 \\
Av. Incontinence episodes & 163 & 4.99 & 0.07 & 0.92 \\
Av. Total voided volume (mL) & 163 & 1140.41 & 13.14 & 167.30 \\
\hline
\end{tabular}

Table 1b - Patient's general characteristics

\begin{tabular}{lccc}
\hline & Male & Female & Total \\
\hline Patients (n) & 94 & 69 & 163 \\
Mean age (yrs) & 30.3 & 61.7 & 43.6 \\
Brain trauma (n) & 59 & 26 & 85 \\
Cerebrovascular accident (n) & 35 & 43 & 78 \\
\hline
\end{tabular}


Table 2 - Correlations of different diary's length with the 7-day duration diary for the evaluated parameters ( $r$ values).

\begin{tabular}{lcccccc}
\hline & Day 1 & Day 2 & Day 3 & Day 4 & Day 5 & Day 6 \\
\hline Micturitions & 0,832 & 0,800 & 0,929 & 0,974 & 0,972 & 0,947 \\
Excreta/micturition & 0,745 & 0,866 & 0,891 & 0,839 & 0,970 & 0,990 \\
Urgency episodes & 0,609 & 0,669 & 0,822 & 0,922 & 0,961 & 0,979 \\
Incontinence episodes & 0,664 & 0,707 & 0,798 & 0,906 & 0,968 & 0,969 \\
\hline Total Urine & 0,823 & 0,955 & 0,956 & 0,902 & 0,973 & 0,980 \\
\hline
\end{tabular}

Table 3-Results of the comparisons between different diary's length (comparison day1 versus day 7 and day 6 versus day 7 were omitted from the Table). Mean difference, $95 \%$ confidence intervals and $P$ value of each comparison are provided.

\begin{tabular}{|c|c|c|c|c|c|}
\hline & & Day 2 vs. Day 7 & Day 3 vs. Day 7 & Day 4 vs. Day 7 & Day 5 vs. Day 7 \\
\hline \multirow{3}{*}{ Micturitions } & Mean diff. & -0.29 & 0.13 & -0.012 & 0.049 \\
\hline & $95 \% \mathrm{Cl}$ & $(-0.522,-0.056)$ & $(-0.014,0.274)$ & $(-0.1,0.075)$ & $(-0.041,0.138)$ \\
\hline & $P$ value & 0.016 & 0.077 & 0.776 & 0.282 \\
\hline \multirow{3}{*}{$\begin{array}{l}\text { Excreta per micturition } \\
\text { (mL/void) }\end{array}$} & Mean diff. & 2.50 & -1.75 & -0.27 & 0.07 \\
\hline & $95 \% \mathrm{Cl}$ & $(0.252,4.757)$ & $(-3.551,0.035)$ & $(-1.956,1.413)$ & $(-0.619,0.778)$ \\
\hline & $P$ value & 0.03 & 0.055 & 0.75 & 0.822 \\
\hline \multirow{3}{*}{ Urgency episodes } & Mean diff. & 0.12 & 0.06 & -0.03 & 0.01 \\
\hline & $95 \% \mathrm{Cl}$ & $(0.013,0.237)$ & $(-0.005,0.126)$ & $(-0.084,0.022)$ & $(-0.021,0.045)$ \\
\hline & $P$ value & 0.028 & 0.071 & 0.254 & 0.489 \\
\hline \multirow{3}{*}{ Incontinence episodes } & Mean diff. & 0.16 & -0.11 & -0.06 & 0.027 \\
\hline & $95 \% \mathrm{Cl}$ & $(0.019,0.306)$ & $(-0.207,-0.031)$ & $(-0.126,0.003)$ & $(-0.009,0.065)$ \\
\hline & $P$ value & 0.027 & 0.044 & 0.061 & 0.141 \\
\hline \multirow{3}{*}{ Voided volume (mL) } & Mean diff. & -12.15 & -9.32 & -8.65 & 4.30 \\
\hline & $95 \% \mathrm{Cl}$ & $(-22.628,-1.633)$ & $(-17.062,0.473)$ & $(-21.402,4.092)$ & $(-0.701,10.304)$ \\
\hline & $P$ value & 0.024 & 0.064 & 0.182 & 0.159 \\
\hline
\end{tabular}


6-day to 7-day duration were omitted from the Table in order to become less complicated. Mean differences along with 95\% confidence intervals and $\mathrm{P}$ values of each comparison, between durations, are presented in the Table.

\section{DISCUSSION}

BD's are widespread used by clinicians in the assessment of voiding dysfunction, providing real-time documentation of LUTS and voiding habits in the patient's environment during everyday life. BD's reduce the recall bias (9-11) that affects questionnaires like history-taking, providing simultaneously objective evidence for the evaluation of several therapeutic interventions. Urodynamic studies (UDS) have been considered as the mainstay in the diagnostic approach of patients suffering from LUTS and cannot be replaced by a diary. Nevertheless, voiding diaries are a useful tool in the initial management of LUTS providing information of daily bladder function, contributing to a more integrated interpretation of the UDS' results, particularly in terms of the functional bladder capacity (12).

No consensus has been reached yet for the optimal duration of BD since no sufficient data could be found in the literature. In several published reports, diaries' duration varies from 24 hours to 14 days. Wyman et al. (9) was the first who recommended that 7-day duration was sufficient to provide reliable documentation of urinary frequency and incontinence episodes in women. The most commonly recommended duration in literature is the 7-day diary $(5,6,9,13-15)$, covering both leisure and working activities. Nevertheless, several studies reported that a diary with less duration than 7 days could furnish comparably reliable results (16).

Nygaard and Holcomb (17) have suggested that a 3-day length is appropriate for trials evaluating stress incontinence, while Brown et al. (18) reported that, in patients suffering from urgency urinary incontinence, a 3-day duration diary could furnish reliable results in terms of daytime frequency, nocturia, urgency and incontinence episodes. In 2005, Dmochowski et al. (19) analyzed, reviewed and compared two large-scale random- ized phase III clinical trials with patients suffering from overactive bladder, treated with transdermal oxybutynin. In both trials patient's symptoms were evaluated through voiding diaries with different durations. In trial A 520 patients documented their symptoms with a 7-day diary, while in trial B 361 patients documented their symptoms with a 3-day diary. Comparing the different durations, they concluded that 3-day diary appears to be equally effective to the 7-day, increasing potentially patient's compliance in clinical trials. Our results appear to be in accordance to the conclusion of the previously mentioned studies. Although the design and the cohort of our study was totally different, our results indicate that a 3-day diary length is adequate, effective and comparable to 7-day, providing credible results in patients suffering from suprapontine pathology in terms of the majority of the evaluated parameters.

Despite protracting the duration of the charts leads to an enhancement of the test reliability, prolonged studies might increase patient's burden leading to decreased compliance, a term described as "diary fatigue" $(6,20)$. Tincello et al. (21) announced that $90.7 \%$ of the patients completed a 3 -day diary compared to only $50 \%$ who completed a 7-day diary, respectively. Shick et al. (16) reported that patient's compliance in BDs follows a logarithmic curve, in contrast to the reliability of the results which follows a bell-shaped curve. The intersection of these two curves represents the minimum duration of a diary which simultaneously provides acceptable reliability of the results.

In 2004, Ku et al. (22) prospectively enrolled 193 patients with incontinence and LUTS in a trial targeting to determine the appropriate duration of a diary assessing patient's compliance and burden. According to their results patient compliance was not compromised by the 7-day diary while patient's burden increases along the increase in the duration of the chart. Although patient's burden was not an evaluated parameter in the design of our study, we should mention the fact that the majority of our patients complained at the second visit about the duration of the BD, claiming that was rather inconvenient.

The majority of previous reports, focusing on the ideal duration of a BD, enrolled patients 
suffering from LUTS caused by several different conditions (diabetes, benign prostate hyperplasia, overactive bladder). In contrast to them, our study population was totally homogenous, since all subjects suffered from neurogenic urinary dysfunction secondary to suprapontine pathology. Thus, the data provided in the literature for this specific population are deficient and sparse. To the best of our knowledge, this is one of the first attempts enrolling such a pure cohort. In such cohort, the neurologic condition of patients is mainly responsible for their urinary dysfunction. As a result, patients' storage and voiding function are less influenced from social, behavioural or environmental factors. The clinical assessment of such patients could be very strenuous due to their neurological pathology and their consequent mobility problems. The majority of patients might be hospitalized for a long time; making their evaluation with the "classic" diary pattern, which includes leisure and working activities, unsuitable. According to our results a 3-day duration bladder diary is efficient and can provide results comparable to a 7-day length for 4 of our evaluated parameters. Only regarding incontinence episodes, 3 days seems inadequate to furnish comparable results, showing a borderline difference. In conclusion, 3-day bladder diary appears to be a quite useful and valuable tool in the evaluation of LUTS in this specific group of patients.

Nevertheless, our study has some limitations. Although it was prospective, we had not designed a second completion of the diaries by the same subjects, as a retest. This could have added concurrent validity to our results (23). Total fluid intake and patient's burden were not evaluated in the study. Moreover, we have to mention that our patients were not evaluated by UDS and that our study was based on the use of a self-recorded diary (20). Finally, we have to point out that the results of the current study cannot be applied to other voiding dysfunctions as only suprapontine neurogenic disorders were included.

\section{CONCLUSIONS}

Three-day duration for bladder diary is as effective and sufficient as the 7-day, in at least
4 out of 5 evaluated parameters, in patients suffering from neurogenic urinary dysfunction due to suprapontine pathology. Thus, a 4-day length $\mathrm{BD}$ can provide comparable results in all parameters. In conclusion, the reliability of results was not compromised by the reduction in the number of days, clearly suggesting that the 7-day diary is unnecessarily protracted. Nevertheless, since LUTS derived from several different clinical conditions, it seems necessary that a validated standardized bladder diary in terms of diary format, duration and evaluated parameters needs to be developed in the future.

\section{CONFLICT OF INTEREST}

None declared.

\section{REFERENCES}

1. Manack A, Motsko SP, Haag-Molkenteller C, Dmochowski $\mathrm{RR}$, Goehring EL Jr, et al. Epidemiology and healthcare utilization of neurogenic bladder patients in a Us claims database. urourol Urodyn. 2011;30:395-401.

2. Gormley EA. Urologic complications of the neurogenic bladder. Urol Clin North Am. 2010;37:601-7.

3. Abrams P, Cardozo L, Fall M, Griffiths D, Rosier P, Ulmsten $\mathrm{U}$, et al. The standardisation of terminology of lower urinary tract function: report from the Standardisation Sub-committee of the International Continence Society. Neurourol Urodyn. 2002;21:167-78.

4. Bright E, Drake MJ, Abrams P. Urinary diaries: evidence for the development and validation of diary content, format, and duration. Neurourol Urodyn. 2011;30:348-52.

5. Abrams P, Klevmark B. Frequency volume charts: an indispensable part of lower urinary tract assessment. Scand J Urol Nephrol Suppl. 1996;179:47-53.

6. Homma Y, Ando T, Yoshida M, Kageyama S, Takei M, Kimoto $\mathrm{K}$, et al. Voiding and incontinence frequencies: variability of diary data and required diary length. Neurourol Urodyn. 2002;21:204-9.

7. Fountoulakis KN, Tsolaki M, Chantzi H, Kazis A. Mini Mental State Examination (MMSE): A validation study in Greece. Am J Alzheimers Dis Other Demen. 2000;15:342-345.

8. Mazurick CA, Landis JR. Evaluation of repeat daily voiding measures in the National Interstitial Cystitis Data Base Study. J Urol. 2000;163:1208-11.

9. Wyman JF, Choi SC, Harkins SW, Wilson MS, Fantl JA. The urinary diary in evaluation of incontinent women: a testretest analysis. Obstet Gynecol. 1988;71:812-7. 
10. Blanker MH, Bohnen AM, Groeneveld FP, Bernsen RM, Prins A, Ruud Bosch JL. Normal voiding patterns and determinants of increased diurnal and nocturnal voiding frequency in elderly men. J Urol. 2000;164:1201-5.

11. Yap TL, Cromwell DA, Brown C, van der Meulen J, Emberton M. The relationship between objective frequency-volume chart data and the I-PSS in men with lower urinary tract symptoms. Eur Urol. 2007;52:811-8.

12. De Wachter S, Wyndaele JJ. Frequency-volume charts: a tool to evaluate bladder sensation. Neurourol Urodyn. 2003;22:638-42.

13. Robinson D, McClish DK, Wyman JF, Bump RC, Fanti JA. Comparison between urinary diaries completed with and without intensive patient instructions. Neurourol Urodyn. 1996;15:143-8.

14. Jaffe JS, Ginsberg PC, Silverberg DM, Harkaway RC. The need for voiding diaries in the evaluation of men with nocturia. J Am Osteopath Assoc. 2002;102:261-5.

15. Locher JL, Goode PS, Roth DL, Worrell RL, Burgio KL. Reliability assessment of the bladder diary for urinary incontinence in older women. J Gerontol A Biol Sci Med Sci. 2001;56:M32-5.

16. Schick E, Jolivet-Tremblay $M$, Dupont $C$, Bertrand PE, Tessier J. Frequency-volume chart: the minimum number of days required to obtain reliable results. Neurourol Urodyn. 2003;22:92-6.

17. Nygaard I, Holcomb R. Reproducibility of the seven-day voiding diary in women with stress urinary incontinence. Int Urogynecol J Pelvic Floor Dysfunct. 2000;11:15-7

18. Brown JS, McNaughton KS, Wyman JF, Burgio KL, Harkaway $\mathrm{R}$, Bergner $\mathrm{D}$, et al. Measurement characteristics of a voiding diary for use by men and women with overactive bladder. Urology. 2003;61:802-9.
19. Dmochowski RR, Sanders SW, Appell RA, Nitti VW, Davila GW. Bladder-health diaries: an assessment of 3-day vs 7-day entries. BJU Int. 2005;96:1049-54.

20. Groutz A, Blaivas JG, Chaikin DC, Resnick NM, Engleman K, Anzalone D, et al. Noninvasive outcome measures of urinary incontinence and lower urinary tract symptoms: a multicenter study of micturition diary and pad tests. J Urol. 2000;164:698-701.

21. Tincello DG, Williams KS, Joshi M, Assassa RP, Abrams KR. Urinary diaries: a comparison of data collected for three days versus seven days. Obstet Gynecol. 2007;109:277-80.

22. Ku JH, Jeong IG, Lim DJ, Byun SS, Paick JS, Oh SJ. Voiding diary for the evaluation of urinary incontinence and lower urinary tract symptoms: prospective assessment of patient compliance and burden. Neurourol Urodyn. 2004;23:331-5.

23. Jimenez-Cidre MA, Lopez-Fando L, Esteban-Fuertes $M$ Prieto-Chaparro L, Llorens-Martinez FJ, Salinas-Casado J, et al. The 3-day bladder diary is a feasible, reliable and valid tool to evaluate the lower urinary tract symptoms in women. Neurourol Urodyn. 2015;34:128-32.
Correspondence address:

Konstantinidis, Charalampos, MD National Institute of Rehabilitation, Urology Chasias Av, 8th bus stop Athens 13122, Greece Telephone: +30 213 201-5311

E-mail: konstantinidischaralampos@yahoo.com 